\title{
Electrolyte beverage consumption alters electrically induced cramping threshold
}

\author{
Jacob E. Earp PhD ${ }^{1}$ | Rebecca L. Stearns PhD ${ }^{2}$ | Andrew Stranieri MS ${ }^{1}$ | \\ James Agostinucci ScD ${ }^{3}$ | Adam S. Lepley PhD ${ }^{4}$ | Taylor Matson BS ${ }^{1}$ | \\ Christie L. Ward-Ritacco PhD ${ }^{1}$
}

${ }^{1}$ Department of Kinesiology, University of Rhode Island, Kingston, Rhode Island

${ }^{2}$ Department of Kinesiology, University of Connecticut, Storrs, Connecticut

${ }^{3}$ Department of Physical Therapy, University of Rhode Island, Kingston, Rhode Island

${ }^{4}$ School of Kinesiology, University of Michigan, Ann Arbor, Michigan

\section{Correspondence}

Jacob E. Earp, 25 West Independence Square, Kingston, RI 02881.

Email: jacob_earp@uri.edu

Funding information

United Citrus Products Corp, Grant/Award Number: AWD 005924

\begin{abstract}
Background: Recent investigations have questioned the role of hydration and electrolytes in cramp susceptibility and thus the efficacy of consuming electrolyte-rich carbohydrate beverages (EB) to control/prevent cramping.

Methods: Nine euhydrated, cramp-prone participants had their cramp susceptibility assessed by measuring the nerve stimulation threshold frequency at which cramping occurs (TF) before and after consumption of an EB (kCal: 120, Na: $840 \mathrm{mg}, \mathrm{K}$ : $320 \mathrm{mg}$, Mg: $5 \mathrm{mg}$ ) and placebo beverage (PB: kCal: 5, Na: $35 \mathrm{mg}$ ). Cramp intensity was assessed using a verbal pain scale and poststimulation electromyography (EMG).

Results: TF was greater in EB $(14.86 \pm 7.47 \mathrm{~Hz})$ than PB $(14.00 \pm 5.03 \mathrm{~Hz} ; P=.038)$ and reported pain was lower in EB $(2.0 \pm 0.6)$ than $\mathrm{PB}(2.7 \pm 0.8 ; P=.025)$ while EMG was similar $(P=.646)$.

Discussion: EB consumption decreased cramp susceptibility and pain but did not prevent cramping in any participants. These results suggest that electrolyte consumption independent of hydration can influence cramp susceptibility in young people.

KEYWORDS

body water, cramp, hydration, sodium, sports drink
\end{abstract}

\section{1 | INTRODUCTION}

Exercise associated muscle cramps (EAMC) are characterized by painful, involuntary muscle contractions during or after fatiguing exercise ${ }^{1}$ and are common in both endurance events ${ }^{2,3}$ and team sports. ${ }^{4}$ The etiology of EAMC has been credited to altered hydration and electrolyte balance stemming from sweat induced sodium loss ${ }^{5-7}$ and altered neurological function related to fatigue. Thus, electrolyte-rich carbohydrate beverages (EB) have the potential to increase EAMC resil-

Abbreviations: $\mathrm{CB}$, control beverage; EAMC, exercise associated muscle cramp; $\mathrm{EB}$, electrolyte-rich beverage; EMG, electromyography; ICC, intra-class correlation; MVIC, maximal voluntary isometric contraction; $\mathrm{PB}$, placebo beverage; TF, threshold frequency at which muscle cramp occurs; VPS, verbal pain scale. ience by offsetting fluid and electrolyte losses and decreasing glycolytic fatigue associated with high intensity exercise. It has been shown that consuming a electrolyte rich carbohydrate beverage in hot environments before exercise more than doubles time to cramping compared with no fluid consumption, ${ }^{8}$ but does not completely prevent cramping from occurring, as $\sim 70 \%$ of participants still experienced cramping. ${ }^{8}$

Several studies have challenged the role that hydration and electrolyte balance have in EAMC by observing that dehydration and serum electrolyte changes during ultra-endurance events were not predictive of runners who experienced EAMC during the race. ${ }^{9,10}$ Rather EAMC was exclusively related to personal cramp history and running intensity. ${ }^{9,10}$ These results suggest that hydration and 
electrolyte balance are not the sole contributors to EAMC and their effects on EAMC susceptibility should be investigated using controlled within subject experiments. There is presently no valid and reliable within subject experimental model to test EAMC susceptibility in the conditions in which they are most likely to occur. Alternatively, the nerve stimulation cramp threshold frequency at which a cramp occurs (TF) has been shown to be a valid and reliable measure of EAMC cramp susceptibility. ${ }^{11,12}$ When using the TF model, it was found that hypohydration did not significantly affect $\mathrm{TF}^{13,14}$; however, it was also observed that TF increased on average $1.8 \mathrm{~Hz}$ after consumption of pickle juice compared with deionized water in hypohydrated participants, supporting consumption of an EB to increase cramp resiliency. ${ }^{15}$

While EB are widely available and commonly consumed during exercise, there is still no well-controlled, randomized control trial that has determined if commercially available EB can decrease cramp susceptibility in euhydrated individuals. Thus, the purpose of the present study is to determine if EB consumption alters the frequency of nerve stimulation at which a cramp occurs (TF) compared with a placebo beverage (PB) with similar fluid volume and flavor profile. We hypothesize that EB consumption will increase TF, indicating greater cramp resilience, when compared with $\mathrm{PB}$ consumption.

\section{2 | METHODS}

This study was a randomized controlled trial with a repeated measures and crossover design (see Supporting Information Figure S1, which is available online). Each participant was considered cramp prone, which was defined in this study as self-reporting experiencing EAMC of the triceps surae on a regular basis (at least monthly). At the time of testing, participants reported that they were free from any musculoskeletal injury or any cardiovascular, neuromuscular, or neurological disease as determined by a medical health history questionnaire. The institutional review board at the University of Rhode Island approved this study and all participants provided written informed consent before participation.

Each participant took part in a screening session used to familiarize them with the experimental methods and ensure that a flexor hallucis brevis cramp could be elicited using electrical stimulation. Participants also completed two experimental sessions, each separated by a 1 -week washout period. ${ }^{12}$ During the experimental sessions, participants had TF of the flexor hallucis brevis measured after consumption of an EB or a PB. When a cramp occurred, muscle activity of the flexor halluces brevis (EMG) was recorded and participants reported the pain experienced during cramping using the verbal pain scale (VPS). ${ }^{16}$ The order of the treatments was randomized and counterbalanced and participants were blinded to treatment.

Each testing session was performed at the same time of day $( \pm 0.5 \mathrm{~h})$. Before testing, sessions participants confirmed they abstained from strenuous physical activity for at least $24 \mathrm{~h}$, alcohol or other depressants for at least $12 \mathrm{~h}$, and caffeine or other stimulants for at least 6 h. Additionally, diet was assessed using a 24-h diet recall.
Diet records were analyzed for calorie and electrolyte consumption using diet analysis software (ASA24, National Institutes of Health, Bethesda, MD). Participants were also instructed to attend sessions euhydrated, which was confirmed as a urine specific gravity $\leq 1.020 .{ }^{17}$ Hydration and fluid distribution were assessed by means of a multifrequency bioelectrical impedance test (InBody 770, InBody, Cerritos, CA). ${ }^{18}$

After hydration, status was assessed and euhydration confirmed, participants had the skin over the medial gastrocnemius and flexor hallucis brevis shaved, abraded, and cleaned with alcohol before being outfitted with 1- $\mathrm{cm}^{2}$ parallel bar wireless EMG electrodes (Trigno IM, Delsys, Natic, USA) over each muscle. Raw EMG signals along with nerve stimulator output signals were recorded by a data acquisition system (PowerLab, AD Instruments, Colorado Springs, $\mathrm{CO}$ ) at a rate of $2000 \mathrm{~Hz}$ and displayed to the participant in real time using associated software (LabChart Pro, AD Instruments, Colorado Springs, CO). Raw flexor hallucis brevis and medial gastrocnemius signals were used for biofeedback to minimize contribution of the plantar flexors during great toe flexion exercise. Flexor hallucis brevis signals were filtered using a Butterworth, 2nd order $10-500 \mathrm{~Hz}$ band-pass filter and reported as a percentage of activity in reference to a maximal voluntary isometric contraction (MVIC). Root mean square of EMG was calculated at rest (1 s), during the MVIC (1 s), and after the onset of the cramp (2 s). EMG during cramping was used to assess cramp intensity and was reported as a percentage of MVIC. ${ }^{19}$

After EMG set-up, the participant was instructed to lay supine on a plinth with their left foot in a custom-built rigging that positioned their ankle to $120^{\circ}$ plantar flexion with their heel and forefoot resting upon a board, and their great toe placed upon a calibrated load cell (see Supporting Information Figure S2). Nylon straps were used to secure the thigh to a plinth, and both nylon and elastic straps were used to secure the leg and foot to the rigging. To prime the nervous system and elicit mild fatigue, participants performed 15, 2-s great toe flexion MVICs each separated by 1 min. ${ }^{15}$ Afterward, participants were given $5 \mathrm{~min}$ of rest followed by 3 additional MVICs each separated by $1 \mathrm{~min}$. Of these $3 \mathrm{MVICs}$, the repetition with the greatest $500 \mathrm{~ms}$ root mean square window was then used to normalize EMG during cramping.

Once the final MVIC was completed, participants were given $0.5 \mathrm{~L}$ of their assigned beverage for consumption. Both beverages were similar volume $(0.5 \mathrm{~L})$, color, and general flavor (lemonade), and mixed with the same brand of bottled water. The EB was a commercially available beverage packet (Fuelocity Plus, United Citrus Corp, Norwood, MA) with $120 \mathrm{kCals}, 29 \mathrm{~g}$ of sugar, $840 \mathrm{mg}$ of sodium, $320 \mathrm{mg}$ of potassium, and $5 \mathrm{mg}$ of magnesium, and $300 \mathrm{mg}$ of L-alanine, while the PB was a commercially available low-calorie beverage packet (Crystal Light, Kraft Foods Inc., Northfield, IL) with $5 \mathrm{kCals}, 0 \mathrm{~g}$ of sugar, $35 \mathrm{mg}$ of sodium, and $0 \mathrm{mg}$ of potassium, magnesium, and L-alanine.

Participants were given up to $5 \mathrm{~min}$ to consume their assigned beverage and were then given $15 \mathrm{~min}$ before the start of TF testing. These durations were selected as they are believed to be similar to those that an athlete may adopt before competition or a conditioning session. During this time, a round 8-mm (diameter) $\mathrm{Ag}-\mathrm{AgCl}$ stimulating electrode was placed over the tibial nerve slightly inferior to the 
medial malleolus, which sent a dispersing electrical stimulus to an $8-\mathrm{cm}^{2}$ sponge electrode placed directly over the lateral malleus. ${ }^{19}$ To ensure proper placement of the electrode the tibial nerve was submaximally stimulated up to three times with an $80 \mathrm{~V}, 2 \mathrm{~ms}$ pulse to ensure strong flexion of the great toe (Grass S88, Astro-Med Inc West Warwick, RI). When positioning was confirmed, electrodes were anchored in place using medical and elastic tape.

To determine TF, participants had their tibial nerve stimulated with an $80 \mathrm{~V}$ biphasic 2 -s train at increasing frequencies until a cramp occurred. The starting frequency for each participant was $8 \mathrm{~Hz}$, and the frequency increased by $2 \mathrm{~Hz}$ every minute until a cramp occurred. Participants who did not cramp after their 12th stimulation $(30 \mathrm{~Hz})$ were excluded from the study, as these participants were determined to not be cramp sensitive. Three criteria were required to be met for a muscle cramp to be confirmed: (1) the participant confirmed that they experienced an involuntary sustained contraction after the cessation of the stimulus, (2) the researcher used load cell data to confirm that involuntary contraction of the flexor hallucis brevis occurred, and (3) the EMG average root mean square for the $2 \mathrm{~s}$ after the cessation of the stimulation had to be greater than the mean plus 2 standard deviations of the raw baseline EMG measurement. ${ }^{19}$ Upon the initiation of a cramp, participants were given $10 \mathrm{~s}$ for the cramp to naturally dissipate. During this time participants remained with their body, foot and toe strapped into position. After $10 \mathrm{~s}$ participants were rapidly unstrapped and they were permitted to stretch and/or massage the toe. Using this method, cramps were reliably induced in both conditions (inter-day intra-class correlation [ICC] = 0.908).

Immediately after this 10 -s period participants were presented with a 6-point VPS, where 1 = No Pain, 2 = Mild Pain, 3 = Moderate Pain, $4=$ Severe Pain, $5=$ Very Severe Pain, and $6=$ Worst Pain Possible. ${ }^{16}$

All results are reported as means and standard deviations. Differences between the two beverages in TF and EMG were determined using a paired t-test and differences in VSP were determined using a Wilcoxon signed rank test. For both tests, the accepted $\alpha$ level of 0.05 was used. Cohen's D effect sizes are reported from delta values. An apriori power analysis revealed that sample size $(n=9)$ of the present study was appropriate to test for a very large effect (Cohen's $D=1.2$ ), which was deemed a clinically significant effect, at a power of 0.85 and accepted alpha of 0.05 using G-Power (Version 3.1, Dusseldorf Germany). Statistical analysis was performed using SPSS (Version 24, IBM, Armonk, NY).

\section{3 | RESULTS}

Twelve cramp-prone participants were recruited and took part in a screening session. Of these participants, three were excluded from the study, as we were unable to successfully induce a cramp by the 12th incremental nerve stimulation $(30 \mathrm{~Hz})$. This resulted in nine participants (seven male and two female, age: $23.6 \pm 4.2$ years, height: $175.4 \pm 16.0 \mathrm{~cm}$, mass: $71.2 \pm 9.3 \mathrm{~kg}$, body composition: $15.5 \pm 9.0 \%$ fat) who completed this study. In addition to regularly experiencing EAMC in their triceps surae, a subset of the cohort also reported regularly experiencing cramps in the foot or toes $(n=5)$, abdominals $(n=2)$, triceps brachii $(n=1)$, and hamstrings $(n=1)$. EAMC were reported to occur during running $(n=5)$, soccer $(n=2)$, basketball ( $n=2)$, lacrosse $(n=1)$, resistance training $(n=1)$, and during the recovery period immediately after intense exercise $(n=4)$. In addition to regularly experiencing EAMC, four participants also reported experiencing spontaneous cramping while sleeping.

When comparing of hydration and fluid distribution before cramp testing it was found that participants had similar urine specific gravity (EB: $1.0119 \pm 0.0056$, PB: $1.0109 \pm 0.00541$, item mean range 0.001) and total body-water (EB: $43.4 \pm 8.5 \mathrm{~kg}, \quad \mathrm{~PB}: \quad 43.9 \pm 9.1 \mathrm{~kg}$, $\mathrm{ICC}=0.996 ; P<0.001)$, intercellular-water (EB: $26.8 \pm 7.3 \mathrm{~kg}, \mathrm{~PB}$ $27.7 \pm 5.9 \mathrm{~kg} ; \mathrm{ICC}=0.916 ; P=0.001$ ), extracellular-water (EB: 16.3 $\pm 2.0 \mathrm{~kg}$, PB: $16.2 \pm 2.2 \mathrm{~kg}$; ICC $=0.997 ; P<0.001$ ), and extracellularwater to total body-water ratio $($ ICC $=0.978 ; P<0.001)$ between conditions.

Analyzed 24-h diet recall revealed calorie, macronutrient and electrolyte consumption did not differ between conditions as indicated by nonsignificant paired t-tests $(P=0.219-0.673)$. Furthermore, electrolyte consumption of potassium (EB: $1377 \pm 459$, PB: $1547 \pm 516 \mathrm{mg}$; ICC $=0.755 ; P=0.038$ ), and magnesium (EB: $233 \pm 78$, PB: 209 $\pm 70 \mathrm{mg}$; ICC $=0.799 ; P=0.017)$ were similar between conditions. However, while not different $(P=0.219)$ consumed sodium was not statistically similar between conditions (EB: $1737 \pm 579$, PB: 1679 $\pm 560 \mathrm{mg}$; ICC $=0.685 ; P=0.058$ ).

Grouped and individual results from cramp testing are reported in Table 1 and Figures 1 and 2, respectively. In the present study, TF was found to be a reliable measure of cramp sensitivity (inter-day reliability: ICC $=0.908$ ). Results demonstrated that TF was significantly higher in EB compared with PB. Compared with the PB, 5 of 9 participants required a greater stimulation frequency to elicit a cramp after consumption of the EB, while the remaining 4 participants had the cramp occur at the same stimulus frequency. Participants reported

\begin{tabular}{|lcclll} 
& \multicolumn{1}{l}{ PB } & EB & $\mathbf{p}$ & $95 \% \mathrm{Cl}$ & Effect Size \\
\hline TF (Hz) & $11.11 \pm 3.89$ & $12.67 \pm 5.10$ & $0.043^{*}$ & 0.06 to 3.05 & 0.800 \\
\hline Pain (VPS) & $2.88 \pm 0.84$ & $2.00 \pm 0.54$ & $0.006^{*}$ & -1.41 to -0.34 & 1.365 \\
\hline EMG (\%MVIC) & $46.2 \pm 22.2$ & $44.1 \pm 19.8$ & 0.724 & -9.6 to 7.0 & 0.122 \\
\hline USG & $1.0109 \pm 0.006$ & $1.0119 \pm 0.005$ & 0.600 & -0.003 to 0.005 & 0.194 \\
\hline
\end{tabular}

TABLE 1 Comparison between testing conditions

Abbreviations: TF, cramp threshold frequency; VPS, verbal pain scale; EMG, electromyography; USG, urine specific gravity; EB, electrolyte rich beverage; PB, Placebo beverage. 
TF

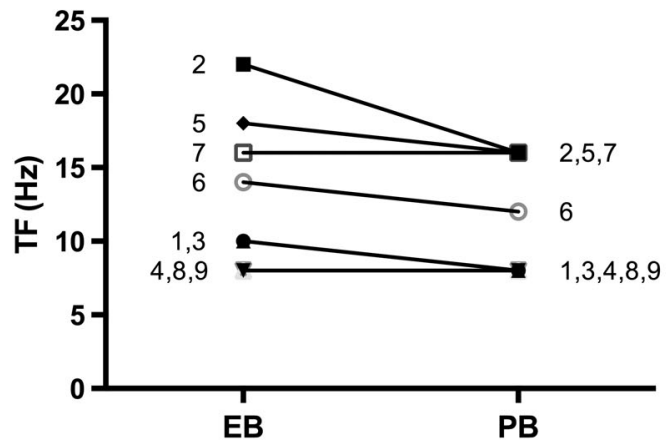

EMG

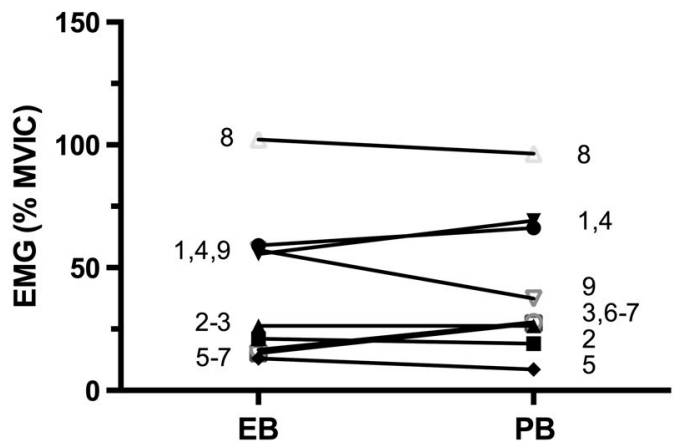

FIGURE 1 Individual responses to consumption of an EB and PB on TF and EMG during electrically induced cramps. Participant numbers (1-9) are provided in spaces next to each measurement

VPS

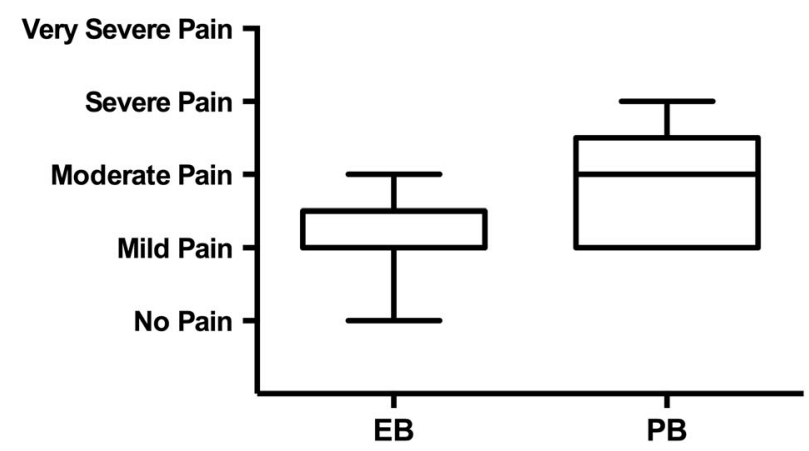

VPS

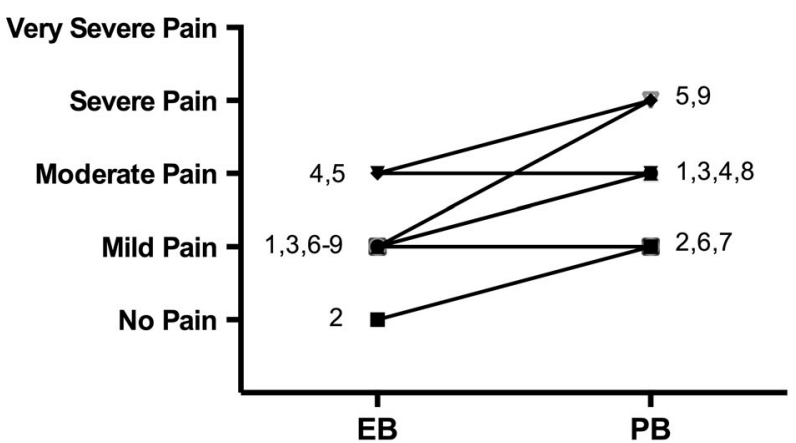

FIGURE 2 Grouped (left) and individual (right) responses to consumption of an EB and PB on reported pain during electrically induced cramps using a VPS. Participant numbers (1-9) are provided in spaces next to each measurement

experiencing significantly greater pain in the PB (Median = Moderate Pain) compared with the EB (Median = Mild Pain) condition when using the verbal pain scale, with 6 of 9 participants reporting less pain in the EB condition and the remaining 3 participants experiencing similar pain. EMG was similar between conditions, with 5 of 9 participants experiencing greater muscle activity after consumption of the EB compared with the PB and 4 of 9 experiencing less muscle activity.

To determine whether an order effect was present, a separate repeated measures ANOVA with Bonferoni correction was used to compare TF between the screening session $(13.3 \pm 7.1)$ and the first $(12.4 \pm 4.9)$ and second $(11.3 \pm 4.2)$ experimental sessions. From this analysis, no difference was observed between the screening session and the first experimental session $(P=1.000)$ or between the first and second experimental session $(P=0.536)$.

To determine if participant condition blinding was effective each participant completed an exit survey asking which session, they believe they received the EB and PB conditions. In this survey, four of nine participants incorrectly guessed their conditions.

\section{4 | DISCUSSION}

The primary finding of the present study was that consumption of an EB resulted in a statistically large increase in TF compared with the consumption of a similarly flavored PB in euhydrated participants. These results indicate that participants required a greater electrical stimulus frequency to elicit a muscle cramp after consumption of the $E B$, which suggests that $E B$ consumption independent of hydration can decrease cramp susceptibility in young people. While this is a statistically significant difference with a large effect size, the clinical applicability of this change in TF remains unknown, as no minimal detectable change in TF has been established in the literature. Additionally, as four of the nine euhydrated cramp prone participants experienced no difference in TF between conditions, it is possible that either the effect size of the EB was smaller than the resolution $(\Delta 2$ $\mathrm{Hz}$ ) of the test or that in these participants there was no effect of beverage on TF. However, the observed difference between conditions of $1.56 \mathrm{~Hz}$ is comparable to differences observed when comparing pickle juice to deionized water $(1.8 \mathrm{~Hz})^{15}$ and TRPV1 and TRPA1 activators (motor neuron inhibitors) to gelatin gel capsules $(1.1 \mathrm{~Hz}){ }^{20}$

Sports drinks, such as the EB used in the present study, have the potential to decrease cramp susceptibility by maintaining fluid and electrolyte homeostasis as a result of the water and electrolyte components, and may increase energy availability during intense exercise, both of which may prevent fatigue. However, the present study design allows for general fluid consumption to be dismissed as a potential mechanism by which TF increased because participants 
(1) attended each session euhydrated (urine specific gravity $\leq 1.020$ ),

(2) had similar hydrations and fluid distribution measures in both testing sessions, and (3) consumed beverages with similar volumes. These results suggest that the proprietary non-water ingredients of the $E B$ accounted for the differences in TF observed presently. However, while general fluid consumption was matched between conditions, it could be reasonable to assume that absorption of the water $15 \mathrm{~min}$ postconsumption was greater in the EB condition than PB condition at this time due to the nonwater ingredients of the EB.

While the effect of the carbohydrate component of the EB on TF cannot be discounted, we believe that an effect is unlikely as the exercise performed as the conditioning activity $(2 \mathrm{~s}$ isometric contractions with $1 \mathrm{~min}$ inter-contraction rest) would suggest that there is minimal contribution of glycolytic metabolism. ${ }^{21}$ However, as carbohydrate mouth rinses have been shown to result in altered exercise performance and brain activity that is independent of gastrointestinal absorption, it is possible that carbohydrate within the EB may have resulted in an altered neural signaling that may have effected TF. ${ }^{22}$

Another factor that could influence TF is the beverage flavor. After the observation that TF was greater after consumption of pickle juice compared with deionized water, it has been suggested that differences between conditions may have results from an oropharyngeal-region neurally mediated reflex. ${ }^{15}$ Although pickle juice has a distinct and contrasting taste compared with deioniozed water, the present study used two beverages with similar flavor profiles to account for a placebo effect and the general effects of flavoring on TF. However, this does not fully account for oropharyngeal-region reflexes as it was impossible to identically match flavor between beverages, and in our exit survey, only four of nine participants incorrectly guessed which condition they received on which day. Therefore, it is possible that participant blinding was not completely effective.

A secondary finding of the present study was that pain was significantly lower in the EB compared with the PB condition, with each participant reporting less (six of nine) or similar (three of nine) pain after consumption of the EB. While significantly less pain was experienced in the EB than PB group, EB also received greater electrical stimulus frequency; therefore, the effect sizes reported for VPS likely underrepresent the true effect of EB on pain. ${ }^{19,23}$ Unlike pain, relative muscle activity did not significantly differ between conditions. These results suggest that the cramping muscle was similarly active between conditions. However, several studies have questioned the use of surface EMG during cramp measurements, as muscle cramp is characterized by spatially inconsistent muscle activation and there exists significant intramuscular cross-talk when measuring the flexor halluces brevis. $^{24}$

While the results from the present study may be of great interest to those who often treat or experience EAMC, it is important to note the following limitations to the study design. First, while strongly related to incidence rate of EAMC, repetitive nerve stimulation does not necessarily incorporate all the factors related to EAMC. Second, intramuscular and skin temperature were not measured, and these variables may affect cramp sensitivity. Third, the homogenous age of the study participants leaves the question as to whether these results would be similar in younger or older cohorts. Additionally, the present study used a single-blinded design rather than a double-blinded design. Finally, while all participants reported they did not have any underling etiology that would predispose them to secondary cramping, it cannot be definitively stated that no such conditions were present in our sample.

Overall, the results of the present study found that EB can increase the electrical stimulus frequency required to elicit a cramp (TF) and decrease the pain experienced when a cramp occurred in euhydrated, cramp-prone individuals. However, EB did not prevent cramps from occurring in any participants. Additionally, the study design used supports the notion that the electrolytes contained within the EB are responsible for such differences; however, differences due to beverage flavoring and carbohydrates cannot be fully discounted. As TF is related to an individual's susceptibility to EAMC, ${ }^{13}$ these results provide support for the efficacy of EBs for increasing resistance to EAMC. However, more research is needed to explore the relationship between electrically induced (TF) and exercise associated (EAMC) muscle cramping as well as to define a clinically significant change in TF.

\section{CONFLICT OF INTEREST}

None of the authors of this study have any professional or personal relationships to the corporate sponsor. The sponsor had no role in data collection, analysis, interpretation, or the dissemination of any results from this study regardless of the findings. The results of the study are presented clearly, honestly, and without fabrication, falsification, or inappropriate data manipulation.

\section{ETHICAL PUBLICATION STATEMENT}

We confirm that we have read the Journal's position on issues involved in ethical publication and affirm that this report is consistent with those guidelines.

\section{REFERENCES}

1. Schwellnus MP, Derman EW, Noakes TD. Aetiology of skeletal muscle "cramps" during exercise: a novel hypothesis. J Sports Sci. 1997;15 (3):277-285.

2. Hiller WD, O'Toole ML, Fortess EE, Laird RH, Imbert PC, Sisk TD. Medical and physiological considerations in triathlons. Am J Sports Med. 1987;15(2):164-167.

3. Laird RH. Medical care at ultra endurance triathlons. Med Sci Sports Exerc. 1989;21(5):S222-S225.

4. Cooper ER, Ferrara MS, Broglio SP. Exertional heat illness and environmental conditions during a single football season in the southeast. J Athl Train. 2006;41(3):332-336.

5. Jahic D, Begic E. Exercise-associated muscle cramp-doubts about the cause. Materia Socio-Medica. 2018;30(1):67-69.

6. Bergeron MF. Muscle cramps during exercise-is it fatigue or electrolyte deficit? Cur Sports Med Repor. 2008;7(4):50-55.

7. Eichner ER. The role of sodium in "heat cramping". Sports Med. 2007; 37(4-5):368-370 
8. Jung AP, Bishop PA, Al-Nawwas A, Dale RB. Influence of hydration and electrolyte supplementation on incidence and time to onset of exercise-associated muscle cramps. J Athl Train. 2005;40(2):71-75.

9. Schwellnus MP, Drew N, Collins M. Increased running speed and previous cramps rather than dehydration or serum sodium changes predict exercise-associated muscle cramping: a prospective cohort study in 210 Ironman triathletes. Br J Sports Med. 2011;45(8):650-656.

10. Schwellnus MP, Nicol J, Laubscher R, Noakes TD. Serum electrolyte concentrations and hydration status are not associated with exercise associated muscle cramping in distance runners. $\mathrm{Br} J$ Sports Med. 2004;38(4):488-492.

11. Miller KC, Knight KL. Electrical stimulation cramp threshold frequency correlates well with the occurrence of skeletal muscle cramps. Muscle Nerve. 2009;39(3):364-368.

12. Stone MB, Edwards JE, Babington JP, et al. Reliability of an electrical method to induce muscle cramp. Muscle Nerve. 2003;27(1):122-123.

13. Braulick KW, Miller KC, Albrecht JM, Tucker JM, Deal JE. Significant and serious dehydration does not affect skeletal muscle cramp threshold frequency. Br J Sports Med. 2013;47(11):710-714.

14. Miller KC, Mack GW, Knight KL, et al. Three percent hypohydration does not affect threshold frequency of electrically induced cramps. Med Sci Sports Exerc. 2010;42(100):2056-2063.

15. Miller KC, Mack GW, Knight KL, et al. Reflex inhibition of electrically induced muscle cramps in hypohydrated humans. Med Sci Sports Exerc. 2010;42(5):953-961.

16. Haefeli M, Elfering A. Pain assessment. Eur Spine J. 2006;15(1):17-24.

17. Kavouras SA. Assessing hydration status. Curr Opin Clin Nutr Metab Care. 2002;5(5):519-524.

18. Sartorio A, Malavolti M, Agosti F, et al. Body water distribution in sever obesity and its assessment from eight-polar bioelectrical impedance analysis. Eur J Clin Nutr. 2005;59(2):155-160.
19. Miller KC, Knight KL. Initial electrical stimulation frequency and cramp threshold frequency and force. J Athl Train. 2012;47(6): 643-647.

20. Behringer M, Nowak S, Leyendecker J, Mester J. Effects of TRPV1 and TRPA1 on the cramp threshold frequency: a randomized, double-blind placebo-controlled trial. Eur J Appl Physiol. 2017;117(8):1641-1647.

21. Painelli VS, Roschel H, Gualano B, et al. The effect of carbohydrate mouth rinse of maximal strength and strength endurance. Eur J Appl Physiol. 2011;111(9):2381-2386.

22. Chambers ES, Bridge MW, Jones DA. Carbohydrate sensing in the human mouth: effects on exercise performance and brain activity. J Physiol. 2009;587(8):1779-1794.

23. Roeleveld K, Van Engelen BG, Stegeman DF. Possible mechanisms of muscle cramp from temporal and spatial surface EMG characteristics. J Appl Physiol. 2000;88(5):1698-1706.

24. Ross $B H$, Thomas CK. Human motor unit activity during induced muscle cramp. Brain. 1995;118(4):983-993.

\section{SUPPORTING INFORMATION}

Additional supporting information may be found online in the Supporting Information section at the end of this article.

How to cite this article: Earp JE, Stearns RL, Stranieri A, et al. Electrolyte beverage consumption alters electrically induced cramping threshold. Muscle Nerve. 2019;60:598-603. https:// doi.org/10.1002/mus.26650 